\title{
原子转移自由基聚合修饰银丝固定化酶反应器的制备 及在蛋白质组鉴定中的应用
}

\author{
周廉淇 ${ }^{1,2}$, 张 姣 ${ }^{2}$, 田 芳 $^{2}$, 张养军 ${ }^{2 *}$, 钱小红 ${ }^{2}$
}

(1. 安徽医科大学, 安徽 合肥 $230032 ; 2$. 蛋白质组学国家重点实验室, 北京蛋白质组研究中心, 军事医学科学院放射与辐射医学研究所, 北京 102206)

摘要: 针对传统溶液酶解存在的酶解时间较长、酶自切物干扰以及蛋白酶不能重复使用等缺陷, 通过电子转移生成 催化剂的原子转移自由基聚合法修饰银丝, 并以其为载体制备了一种新型的固定化酶反应器。用质谱考察了银丝 固定化酶反应器 (SW-Trypsin) 的酶解效率、重复性和回收率。结果表明: 线毛状聚合物修饰的 SW-Trypsin 的酶解 效率较高, 酶解标准蛋白牛血清白蛋白 (BSA) $20 \mathrm{~min}$ 后, 肽段的氨基酸序列覆盖率可达 $93 \%$, 高于传统溶液酶解方 法酶解 $16 \mathrm{~h}$ 所得 79\% 的覆盖率。使用该固定化酶反应器于一个月内 8 次酶解 BSA 所得的氨基酸序列覆盖率在 $89 \%$ 到 $97 \%$ 之间, 平均覆盖率为 $94 \%$, 显示出良好的稳定性。另外, 该固定化酶反应器酶解马心肌红蛋白 (MYO) 的回收率为 $87.67 \%$ 。最后, 用 SW-Trypsin 酶解腾冲嗜热菌全蛋白 $20 \mathrm{~min}$, 所鉴定到的氨基酸序列覆盖率和蛋白 数量与同样条件下溶液酶解 $16 \mathrm{~h}$ 的结果接近, 且零漏切位点肽段的比例更高。加之容易分离的优点, SW-Trypsin 在蛋白质组学的应用中具有良好的前景。

关键词:原子转移自由基聚合反应;蛋白质组学;质谱;固定化酶反应器; 银丝

中图分类号: O658 文献标识码:A 文章编号: 1000-8713(2013)04-0355-07

\section{Preparation of a trypsin immobilized reactor on silver wire modified by atom transfer radical polymer and its application in proteome identification}

\author{
ZHOU Lianqi ${ }^{1,2}$, ZHANG Jiao $^{2}$, TIAN Fang ${ }^{2}$, ZHANG Yangjun ${ }^{2 *}$, QIAN Xiaohong ${ }^{2}$ \\ (1. Anhui Medical University, Hefei 230032, China; 2. State Key Laboratory of Proteomics, \\ Beijing Proteome Research Center, Beijing Institute of Radiation Medicine, Beijing 102206, China)
}

Abstract: The routine proteolysis of proteins is performed in solution, but it suffers from drawbacks such as long incubation time, enzyme autodigestion, and non-reusability. Therefore we here demonstrated that trypsin could be immobilized on silver wire modified by atom transfer radical polymerization to prepare a new kind of enzyme immobilized reactor. The digestion efficiency, repeatability and recovery of the silver wire-trypsin reactor ( SW-Trypsin) were evaluated by mass spectrometry ( MS) analysis. Highly efficient digestion was achieved by using SW-Trypsin within only $20 \mathrm{~min}$. Standard protein could be almost completely digested with sequence coverage up to $93 \%$, which is higher than that of $79 \%$ sequence coverage obtained by in-solution digestion for $16 \mathrm{~h}$. Bovine serum albumin (BSA) was digested eight times within a month by using the SW-Trypsin. The results of sequence coverage were between $89 \%$ to $97 \%$, with an average sequence coverage of 94\%, which showed that SW-Trypsin had good stability. In addition, the recovery test by using myoglobin ( MYO) showed that the recovery rate was 87. 67\%. At last, the extract from Tengchong thermophilic bacteria was digested by SW-Trypsin in $20 \mathrm{~min}$ and in-solution trypsin in $16 \mathrm{~h}$. The results of sequence coverages and the number of

*通讯联系人. E-mail: zhangyangjun6314@ yahoo. com. cn.

基金项目: 国家重大科学计划项目 (2012CB910603,2010CB912704); 国家重大科学仪器设备开发专项项目 (2011 YQ030139, 2011YQ06008408,2012YQ12004407); 国家高技术研究发展计划项目 (2012AA020202); 国家自然科学基金项目 $(20735005,21275159)$.

收稿日期: 2012-11-05 
identified proteins were similar. Moreover, the ratio of the number of peptides with zero missed cleavages to the number of all identified peptides by using SW-Trypsin was higher than that by in-solution digestion. Also, the SW-Trypsin was easily removed from the digestion solution. Good performances of SW-Trypsin implied that it has a good prospect in the application in future proteomics research.

Key words : atom transfer radical polymerization; proteomics ; mass spectrometry ( MS) ; trypsin immobilized reactor; silver wire

对生物系统中蛋白质组进行研究可为更好地理 解复杂的生物机制提供有用信息 ${ }^{[1]}$ 。而在蛋白质 组学研究中最常用的是 “bottom-up” 研究策略, 其 主要有两种工作流程:一种是先在蛋白质水平上分 离, 再酶解后采用肽质量指纹谱 ${ }^{[2]}$ 或采用串联质谱 分析 ${ }^{[3]}$; 而另一种则是全蛋白在不经过任何分离的 情况下直接酶解后产生多肽混合物, 该混合物经过 多维色谱分离后采用串联质谱进行分析,将其产生 的串联质谱数据通过搜库软件对蛋白质进行鉴定， 该方法又常被称为 “鸟枪法” ${ }^{[4]}$ 。鉴于上述两种主 要的工作流程中都需要将蛋白质酶解后对其酶解产 物进行质谱 $(\mathrm{MS})$ 分析, Bruening 等 ${ }^{[5]}$ 就曾在文章 中表示蛋白质的鉴定及其翻译后修饰的研究中最常 用的方法是将蛋白质酶解后对其酶解产物进行 MS 分析,蛋白质的酶解在蛋白质组学的研究中发挥着 关键作用。

由于胰蛋白酶可以特异性地切断精氨酸或赖氨 酸羧基端且酶解形成的肽段的相对分子质量适合于 质谱的质量鉴定范围 ${ }^{[6]}$, 所以胰蛋白酶成为蛋白质 组学研究中最常使用的蛋白酶 ${ }^{[7]}$ 。传统的酶解方 法是将胰蛋白酶溶解在溶液中使用; 为防止胰蛋白 酶自身酶解带来的干扰肽段 ${ }^{[8]}$, 通常只加人少量的 胰蛋白酶, 因此需要较长的酶解时间 ${ }^{[9]}$, 这就成为 高通量蛋白质组学研究中的限速环节 ${ }^{[10]}$ 。为了解 决溶液酶解过程中存在的问题,蛋白质组学研究中 引进并发展了固定化酶技术。固定化酶技术由于其 稳定性好、可重复使用、易于分离等优势受到了越来 越多的关注 ${ }^{[11]}$ 。目前已有很多关于固定化酶反应 器的报道, 其材料也多种多样, 包括纳米金颗 粒 ${ }^{[12-14]}$ 、包裹二氧化硅的磁性纳米颗粒 ${ }^{[15,16]}$ 、氧化 石 墨烯 ${ }^{[17]}$ 、芯片材 料 $^{[18,19]}$ 、棉纱 ${ }^{[20]}$ 、介孔 材 料 ${ }^{[21,22]}$ 、石英毛细管整体柱 ${ }^{[23,24]}$ 以及杂化整体 柱 ${ }^{[25]}$ 等,但绝大部分的载体都会有其缺陷。我们在 冯娟娟等 ${ }^{[26]}$ 的工作基础上, 发展了一种制备简单、 容易分离的金属丝固定化酶反应器,采用原子转移 自由基聚合反应(ATRP $)^{[27]}$ 生成带有环氧基团的 聚合物链, 并使用环氧基固定胰蛋白酶。传统的
ATRP 反应中的催化剂对 $\mathrm{O}_{2}$ 及 $\mathrm{H}_{2} \mathrm{O}$ 较为敏感, 不 利于反应的控制; 在本研究中, 采用了电子转移生成 催化剂的原子转移自由基聚合 (AGET-ATRP ${ }^{[28]}$ 的 方法在银丝表面修饰上含有环氧基的聚合物长链, 制备了一种极易分离的固定化酶反应器, 并将其初 步应用于腾冲嗜热菌蛋白质组的鉴定。

\section{1 实验部分}

\section{1 仪器与试剂}

TSQ Vantage 三重四极杆质谱仪 (美国 Thermo Fisher Scientific 公司 ); 4800 基质辅助激光解 吸电离飞行时间串联质谱仪 (MALDI-TOF MS, 美 国 AB SCIEX 公司); 反相高效液相色谱-电喷雾-线 性离子阱-傅里叶变换离子回旋共振质谱仪 (RPLCESI-LTQ-ICR-FT MS, 美国 Thermo Fisher Scientific 公司), Eksigent nanoLC-2D 液相色谱系统(荷 兰 Eksigent 公司); 安捷伦 1100 毛细管色谱系统 (美国 Agilent 公司)；3K30 型高效离心机 (美国 SIGMA 公司); SC100A Speedvac Plus 真空冰冻干 燥机（美国 Thermo Savant 公司); Sartorius CPA225D 分析天平 (德国 Sartorius 公司); JY92II 超声细胞粉碎机 ( 宁波新芝生物科技股份有限公 司); Thermo Heraeus Fresco 21 微量离心机(德国 Heraeus 公司) ; NanoDrop 2000c 超微量分光光度 计(美国 Thermo Fisher Scientific 公司)。

主要试剂: 马心肌肌红蛋白 (MYO)、牛血清白 蛋白 (BSA)、 $\alpha$-氧基-4-羟基肉桂酸 ( CHCA)、甲酸 (FA)、三甲基丙烯酸缩水甘油酯( GMA)、11-颈基1-十一烷醇和 $N, N, N^{\prime}, N^{\prime \prime}, N^{\prime \prime}$-五甲基二乙烯三胺 均购自于美国 Sigma 公司; 1,4-二硫苏糖醇 (DTT)、碘乙酰胺 (IAA) 和胰蛋白酶购自美国 Promega 公司; 色谱级乙腈 (ACN) 购自美国 J. T. Baker 公司; 氧基硼氢化钠 ( sodium cyanoborohydride) 购自美国 Acros 公司; 尿素和三羟甲基胺 基甲烷 (Tris) 购自美国 Affymetrix 公司; 其余试剂 均购自北京化工厂; 所有实验用水由 Milli-Q 纯水系 统提供(美国 Millipore 公司)。 


\section{2 实验方法}

\subsection{1 银丝的前处理}

截取约 $8 \mathrm{~cm}$ 长的银丝,绕成弹簧状后置于 1.5 $\mathrm{mL}$ eppendorf (EP) 管中,使用 $25 \%$ (体积分数) 的 硝酸去除表面的氧化膜。之后用水清洗 3 次,再用 乙醇清洗 3 次, 置于室温干燥后备用。

\subsection{2 银丝固定化酶反应器 (SW-Trypsin) 的制备}

我们对文献 ${ }^{[29,30]}$ 中报道的合成引发剂的方法 略作改动。将原来室温反应完成后提取及浓缩的步 骤改为直接使用滤纸过滤。滤液用 $\mathrm{N}_{2}$ 吹干至略有 粘滞感, 溶解在无水乙醇中, 配制成终浓度为 10 $\mathrm{mmol} / \mathrm{L}$ 的反应液。取一根银丝置于含 $500 \mu \mathrm{L}$ 反 应液的 $\mathrm{EP}$ 管中, 置于旋转混合仪上过夜反应。用 甲醇清洗掉剩余引发剂后, 加人 ATRP 反应液(含 2 mol/L GMA 、0. $02 \mathrm{~mol} / \mathrm{L} \mathrm{CuCl} 、 0.03 \mathrm{~mol} / \mathrm{L} N, N$, $N^{\prime}, N^{\prime \prime}, N^{\prime \prime}$-五甲基二乙烯三胺和 $0.001 \mathrm{~mol} / \mathrm{L} \mathrm{CuCl}_{2}$ 的环己醇溶液), 再加人 $0.03 \mathrm{~mol} / \mathrm{L}$ 葡萄糖, 室温振 荡反应 $24 \mathrm{~h}$ 。后续反应参见相关文献 ${ }^{[15,16]}$ 。 $\mathrm{SW}$ Trypsin 制备完成后,使用 $50 \mathrm{mmol} / \mathrm{L}$ 的 Tris- $\mathrm{HCl}$ 缓冲液清洗, $4{ }^{\circ} \mathrm{C}$ 保存。

在固定胰蛋白酶前, 采用超微量分光光度计测 定胰蛋白酶反应液的蛋白浓度; 固定后,重新测定该 反应液的浓度,计算得到 SW-Trypsin 的负载量。

\subsubsection{SW-Trypsin 酶解标准蛋白}

配制 $2 \mathrm{~g} / \mathrm{L}$ 的 BSA 和 $1 \mathrm{~g} / \mathrm{L}$ 的 MYO 溶液 (溶 剂为 $50 \mathrm{mmol} / \mathrm{L}$ Tris- $\mathrm{HCl}$ 缓冲液 )。 $\mathrm{BSA}$ 溶液中加 人终浓度为 $10 \mathrm{mmol} / \mathrm{L}$ 的 DTT, 置于沸水中加热变

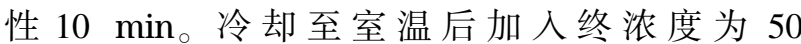
$\mathrm{mmol} / \mathrm{L}$ 的 IAA, 暗处放置 $1 \mathrm{~h}$, 补加 DTT, 于 $37{ }^{\circ} \mathrm{C}$ 水 浴酶解 $20 \mathrm{~min}$ 。将 SW-Trypsin 取出, 溶液稀释后 点靶进行 MALDI-TOF MS 分析。由于 MYO 不含 半胱氨酸, 不存在二硫键, 故直接置于沸水中进行热 变性, 再用 SW-Trypsin 于 $37{ }^{\circ} \mathrm{C}$ 酶解 $20 \mathrm{~min}$ 。将 SW-Trypsin 取出, 溶液稀释后点靶进行 MALDITOF MS 分析。

分别采用 SW-Trypsin 酶解和传统溶液酶解 MYO,使用 Eksigent nanoLC-2D-TSQ Vantage 联用 系统对两种条件下酶解后所得的 3 条零漏切位点肽 段（VEADIAGHGQEVLIR， HGTVVLTALGGILK， ALELFR) 进行分析,用 Pinpoint 软件处理数据。

\subsection{4 腾冲啫热菌全蛋白的提取}

将腾冲嗜热菌干粉溶于 $1 \mathrm{~mL}$ 裂解液 $(8 \mathrm{~mol} / \mathrm{L}$ 尿素,50 mmol/L Tris- $\mathrm{HCl})$ 中, 在冰浴下进行超声 提取。超声功率为 $400 \mathrm{~W}$, 脉冲工作模式:工作 $1 \mathrm{~s}$, 间隔 $2 \mathrm{~s}$, 工作 $60 \mathrm{~s}$ 后静置 $3 \mathrm{~min}$, 重复以上操作 8
次。于 $4{ }^{\circ} \mathrm{C}$ 下以 $14000 \mathrm{r} / \mathrm{min}$ 离心 $1 \mathrm{~h}$, 上清液即为 全蛋白提取液。使用分光光度计测定全蛋白浓度, 分装后保存于 $-80{ }^{\circ} \mathrm{C}$ 冰箱备用。

\subsubsection{SW-Trypsin 酶解腾冲嗜热菌全蛋白}

取 $100 \mu \mathrm{g}$ 的腾冲嗜热菌全蛋白,加人终浓度为 $10 \mathrm{mmol} / \mathrm{L}$ 的 DTT, 于 $37{ }^{\circ} \mathrm{C}$ 水浴中还原 $4 \mathrm{~h}$, 冷却 至室温, 加人终浓度为 $50 \mathrm{mmol} / \mathrm{L}$ 的 IAA, 暗处放 置 $1 \mathrm{~h}$ 进行烷基化。补加 DTT 后使用 $50 \mathrm{mmol} / \mathrm{L}$ Tris-HCl 缓冲液稀释至终体积为 $200 \mu \mathrm{L}$ 。将其分 为两份, 一份采用传统溶液酶解方法酶解 $16 \mathrm{~h}$; 另 外一份加人 SW-Trypsin, 于 $37{ }^{\circ} \mathrm{C}$ 水浴 $20 \mathrm{~min}$, 然后 将 SW-Trypsin 取出。两种方法酶解完成后各取 $0.5 \mu \mathrm{g}$ 酶解肽段,脱盐后用 RPLC-ESI-LTQ-ICR-FT MS 进行分离和鉴定。

\subsection{6 色谱及质谱条件}

MALDI-TOF MS 条件: 仪器控制软件为 4000 Series Explorer ${ }^{\mathrm{TM}}$ software。用 MYO 的胰蛋白酶 酶解肽段作为标准对仪器进行外标校正, 要求相对 标准偏差小于 $10 \times 10^{-6}$ 。一级质谱数据采集使用 MS-2 $\mathrm{kV}$ 反射模式, 加速电压为 $20 \mathrm{kV}$ 。MYO 的酶 解产物扫描范围: $m / z 620 \sim 4000 ; \mathrm{BSA}$ 的酶解产 物扫描范围: $m / z 650 \sim 4000$ 。激光能量调整合适, 每张谱图由 1600 个亚谱累加获得。分别取两种标 准蛋白的酶解溶液稀释到 $0.01 \mathrm{~g} / \mathrm{L}$, 各取 $0.8 \mu \mathrm{L}$ 点 到 MALDI-TOF MS 的靶上, 待室温干燥后, 点上 $0.8 \mu \mathrm{L} \mathrm{CHCA}$ 基质结晶后进行 MALDI-TOF MS 分 析。所得质谱数据采用 MASCOT 检索软件 ${ }^{[31]}$ 进行 肽质量指纹图谱分析。

Eksigent nanoLC-2D-TSQ Vantage 联用系统 条件: 自动进样器上样, 上样量为 $0.01 \mu \mathrm{g}$ 。液相色 谱条件为: 流动相 $\mathrm{A}$ 为含 $0.1 \%$ (体积分数, 以下同 此) $\mathrm{FA}$ 和 $2 \% \mathrm{ACN}$ 的水溶液, 流动相 $\mathrm{B}$ 为含 $0.1 \%$ $\mathrm{FA}$ 和 98\% $\mathrm{ACN}$ 的水溶液。洗脱条件为:0 1 min, $5 \% \mathrm{~B} \sim 8 \% \mathrm{~B}$; $1 \sim 26 \min , 8 \% \mathrm{~B} \sim 35 \% \mathrm{~B}$; $26 \sim 28$ $\min , 35 \% \mathrm{~B} \sim 95 \% \mathrm{~B} ; 28 \sim 33 \min , 95 \% \mathrm{~B} ; 33 \sim 35$ $\mathrm{min}, 100 \% \mathrm{~A}$ 平衡色谱柱。流速为 $380 \mathrm{~nL} / \mathrm{min}^{\circ}$ 。质 谱条件:正离子模式,喷射电压为 $1900 \mathrm{~V}$, 毛细管温 度为 $240{ }^{\circ} \mathrm{C}$; 采用 $\mathrm{SRM}$ 扫描模式: $\mathrm{Q} 1$ 的分辨率设置 为 $0.7(\mathrm{FWHM}), \mathrm{Q} 2$ 的氩气碰撞压力为 1.5 $\mathrm{mTorr}, \mathrm{Q} 3$ 的分辨率设置为 0.7 ( FWHM) ; 循环时 间为 $2 \mathrm{~s}$; 质谱采集时间为 $40 \mathrm{~min}$ 。

RPLC-ESI-LTQ-ICR-FT MS 条件: 自动进样器 上样, 上样量为 $20 \mu \mathrm{L}(0.5 \mu \mathrm{g})$ 。在 Agillent 1100 系统上进行色谱分离, 洗脱的馏分经 ESI 离子源离 子化后进人质谱进行分析。液相色谱条件为: 流动 
相 $\mathrm{A}$ 为含 $0.1 \% \mathrm{FA}$ 和 $2 \% \mathrm{ACN}$ 的水溶液, 流动相 $\mathrm{B}$ 为含 $0.1 \% \mathrm{FA}$ 和 $80 \% \mathrm{ACN}$ 的水溶液。洗脱条件 为: $0 \sim 1 \mathrm{~min}, 0 \% \mathrm{~B} \sim 6 \% \mathrm{~B} ; 1 \sim 91 \mathrm{~min}, 6 \% \mathrm{~B} \sim$ 40\% B; $91 \sim 101 \mathrm{~min}, 40 \% \mathrm{~B} \sim 100 \% \mathrm{~B} ; 101 \sim 111$ $\min , 100 \% \mathrm{~B}$; $111 \sim 112 \min , 100 \% \mathrm{~B} \sim 100 \% \mathrm{~A}$; $112 \sim 120 \mathrm{~min}, 100 \% \mathrm{~A}$ 平衡色谱柱。流速为 300

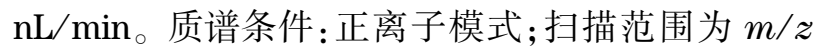
$375.0 \sim 1500.0$; 质谱采集时间为 $110 \mathrm{~min}$; 数据依 赖模式为选取一级质谱全扫描的 10 个信号最高的 离子进行 MS/MS 分析; 碰撞能量为 $35 \mathrm{~V}$, 活化时间 为 $10 \mathrm{~ms}$; 重复采集谱图 2 次后, 动态排除, 时间为 $65 \mathrm{~s} 。 \mathrm{MS} / \mathrm{MS}$ 谱图数据检索用 $\mathrm{pFind}^{[32]}$ 软件分析, 具体设置包括:蛋白质水解酶为胰蛋白酶; 允许漏切 位点数 2 个; 固定修饰为半胱氨酸烷基化; 可变修饰 为甲硫氨酸氧化; 母离子质量偏差为 $20 \mathrm{ppm}$

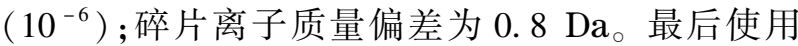
pBuild 软件对搜库结果进行过滤，使得总体的假阳 性率小于 $1 \%$

\section{2 结果与讨论}

\subsection{SW-Trypsin 的制备与表征}

SW-Trypsin 的制备流程如图 1 所示。首先, 依 靠引发剂上的颈基将引发剂连接到银丝表面,使之 与 GMA 单体进行 AGET-ATRP 聚合反应。即在银 丝表面固定大量的环氧基,再采用乙二胺与 GMA 进行开环反应使其带上氨基, 最后用戊二醛交联表 面的氨基和胰蛋白酶的氨基而将胰蛋白酶固定到银 丝表面。
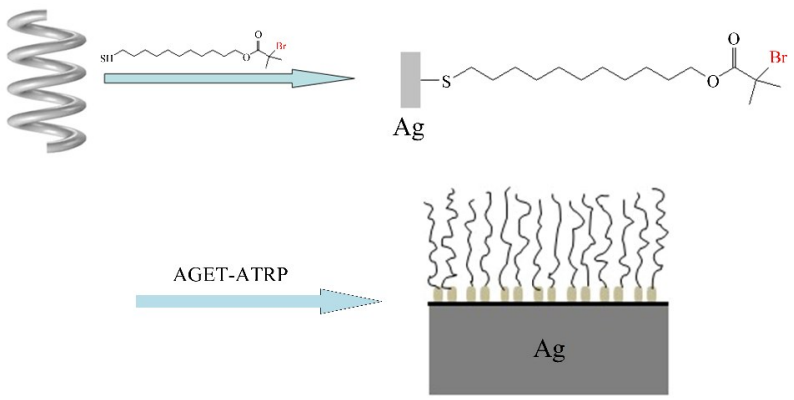

图 1 SW-Trypsin 的制备流程图

Fig. 1 Flow chart for the preparation procedures of silver wire-trypsin reactor (SW-Trypsin)

AGET-ATRP 反应后的银丝有肉眼可见的聚合 物层, 利用光电显微镜对聚合物链修饰的银丝以及 未经任何处理的银丝进行表面结构表征。从图 2 中 可看出,仅 40 倍放大即可观察到经 AGET-ATRP 反 应后银丝表面生成大量均匀的毛发状聚合物修饰， 无明显可见的裸露银丝。毛发状聚合物的修饰提高
了银丝的比表面积, 使银丝修饰了高密度的环氧基 团, 大幅提高了固定化胰蛋白酶的负载量, 进而通过 增加酶量而提高酶解效率。
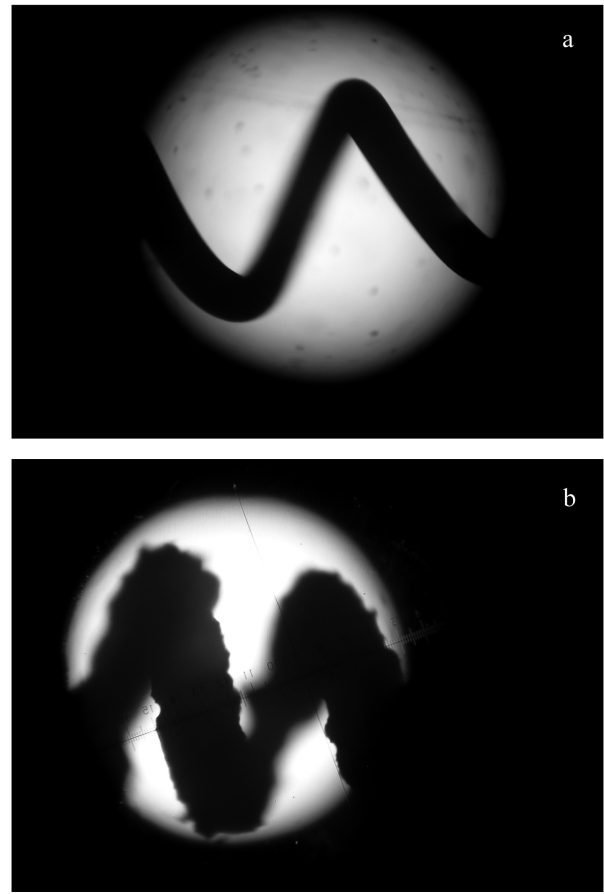

图 2 (a) 未经任何处理的银丝和 (b) 毛发状聚合物包裹的 银丝 (SW-Trypsin) 的光电显微镜图 $(40 \times)$

Fig. 2 Microscope images of $(a)$ silver wire $(40 \times)$ and (b) SW-Trypsin $(40 \times)$

为进一步考察其负载量, 使用超微量分光光度 计测定胰蛋白酶固定前后溶液中 $A_{280}$ 处吸收峰变 化, 计算出浓度差。再根据反应液的体积为 $0.5 \mathrm{~mL}$ 计算出一根 $8 \mathrm{~cm}$ 长的银丝的胰蛋白酶负载量。平 行试验重复测定 3 次的结果列于表 1 中。胰蛋白酶 固定前后平均质量浓度差为 $0.732 \mathrm{~g} / \mathrm{L}$, 负载量为 $0.366 \mathrm{mg}$, 即每一根 $8 \mathrm{~cm}$ 长的银丝可固定 0.366 $\mathrm{mg}$ 胰蛋白酶。AGET-ATRP 生成的聚合物链上具 有的高密度环氧基增加了胰蛋白酶的负载量。

表 1 胰蛋白酶固载量考察

Table 1 Amount of trypsin immobilized on the silver wire

\begin{tabular}{cc}
\hline \multicolumn{2}{c}{$\rho($ Trypsin $) /(\mathrm{g} / \mathrm{L})$} \\
\hline Before immobilization & After immobilization \\
\hline 1.874 & 1.127 \\
1.861 & 1.148 \\
1.874 & 1.138 \\
\hline
\end{tabular}

\subsection{SW-Trypsin 的酶解效率考察}

我们选取了两种标准蛋白质考察 SW-Trypsin 的酶解效率。一种是被认为对蛋白酶的水解作用具 有耐受性的 $\mathrm{MYO}^{[33]}$, 其相对分子质量为 16900 $\mathrm{Da}$; 另一种是 $\mathrm{BSA}$, 其相对分子质量为 $66430 \mathrm{Da}$, 
由 583 个氨基酸组成,其中 35 个半胱氨酸形成 17 个二硫键,在肽链的第 34 位有一自由颈基。两种蛋 白质都分别采用 SW-Trypsin 和传统的溶液酶解方 法进行酶解,其中 SW-Trypsin 酶解两种蛋白质仅需 $20 \mathrm{~min}$,而传统的溶液酶解则需要 $16 \mathrm{~h}$ 。

MYO 和 BSA 经 SW-Trypsin 酶解后进行 MALDI-TOF MS 分析的一级质谱图分别如图 3 和图 4 所示。SW-Trypsin 酶解 MYO 和 BSA 的氨基酸序 列覆盖率分别可以达到 $98 \%$ 和 $93 \%$ 。而传统的蛋 白质溶液酶解所得氨基酸覆盖率仅分别为 $88 \%$ 和 $79 \%$,如表 2 所示。

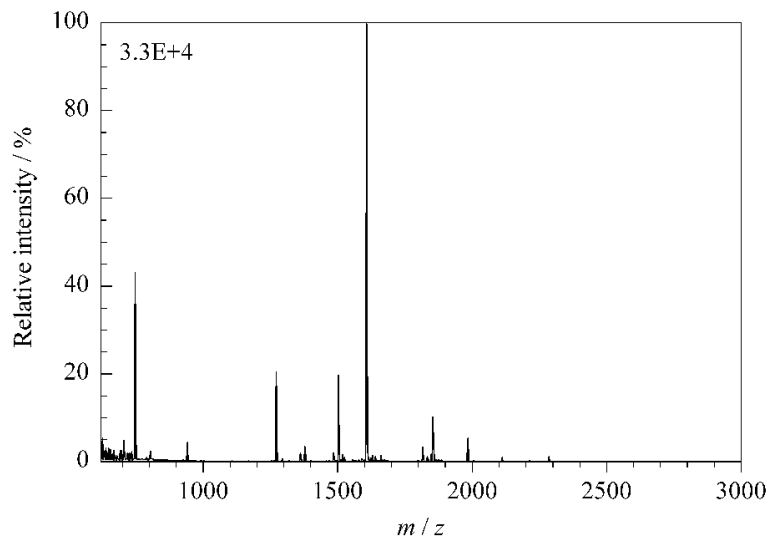

图 3 SW-Trypsin 酶解 MYO 的 MALDI-TOF MS 谱图

Fig. 3 MALDI-TOF mass spectrum of peptides of MYO digested with SW-Trypsin

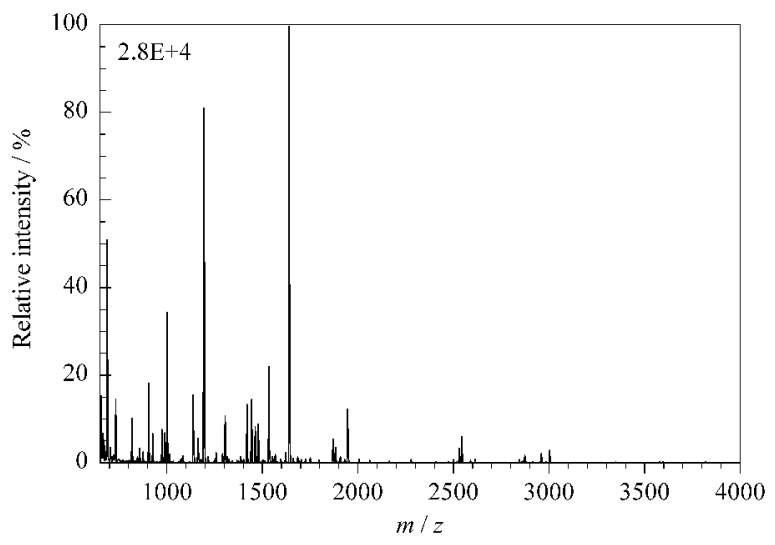

图 4 SW-Trypsin 酶解 BSA 的 MALDI-TOF MS 谱图

Fig. 4 MALDI-TOF mass spectrum of peptides of BSA digested with SW-Trypsin

表 2 传统溶液酶解与 SW-Trypsin 酶解 MYO 和 BSA 所得 氨基酸序列覆盖率比较

Table 2 Comparison of sequence coverages between traditional in-solution digestion and SWTrypsin digestion of MYO and BSA MYO

Method

Number of Sequence identified coverage/ peptides $\%$

23

28

88

98

\section{BSA}

Number of Sequence identified coverage/ peptides $\%$
进一步研究了两种蛋白质的肽段鉴定数量。传 统溶液酶解 MYO 和 BSA 后, 经 MALDI-TOF MS 分 析,分别鉴定到 23 条和 63 条肽段。而 MYO 和 BSA 经 SW-Trypsin 酶解后, 分别鉴定到 28 条和 104 条 肽段, 肽段鉴定数量有所提高, 这有助于降低蛋白质 鉴定的假阳性率。

当传统溶液酶解方法用于 ${ }^{18} \mathrm{O}$ 标记时, 常常需 要通过变性、加热和酸化等方法来避免回交换反应, 而 SW-Trypsin 完成酶解后可直接使用镊子夹出。 另外,SW-Trypsin 相对溶液酶解具有酶解效率高的 优点。由于银丝表面通过 AGET-ATRP 反应生成的 聚合物链上带有高密度环氧基, 从而使其固定上大 量的胰蛋白酶,而且非交联的聚合物链有利于酶与 底物的接触。SW-Trypsin 酶解效率接近磁性纳米 颗粒, 制备的步骤也相似, 但制备完成后不存在团聚 问题, 且分离相对容易; 酶解完成后的分离比纳米金 颗粒容易; 酶解样品的通量明显优于石英毛细管和 芯片材料;较整体柱的制备更为容易。

\section{$2.3 \mathrm{SW}-$ Trypsin 的稳定性与回收率考察}

为考察该固定化酶反应器的稳定性, 即是否可 以重复使用,用一根 SW-Trypsin 在一个月内酶解 8 份 BSA, 酶解后的产物分别进行 MALDI-TOF MS 分 析。质谱数据用 MASCOT 检索软件进行肽质量指 纹图谱分析,所得氨基酸序列覆盖率在 89\% 到 97\% 之间,平均覆盖率为 $94 \%$, 相对标准偏差 (RSD) 为 $3.13 \%$, 如图 5 所示。说明 SW-Trypsin 能够多次重 复使用,具有较好的稳定性。

考虑到实验中生成的聚合物链可能导致的非特 异性吸附,我们还使用 MYO 对该固定化酶的回收 率进行了考察。通过选择性反应监测的方法分别对 经 SW-Trypsin 酶解和传统溶液酶解后所得的零漏 切位点肽段进行定量, 最终得到该固定化酶的回收

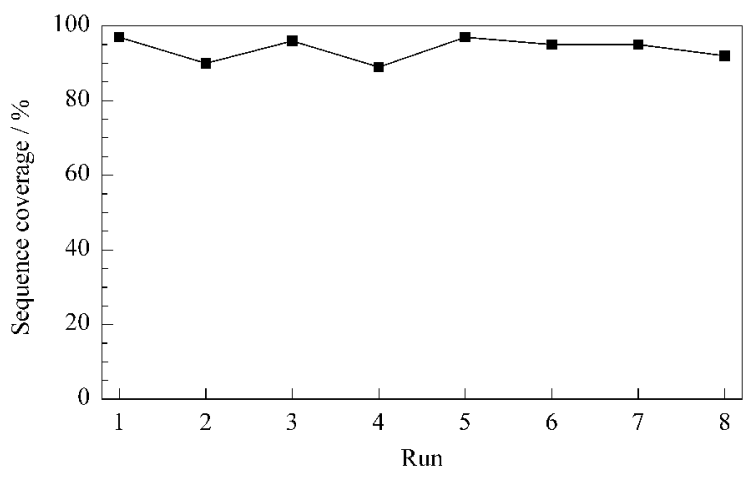

图 $5 \mathrm{SW}$-Trypsin 酶解 BSA 稳定性和重现性考察 Fig. 5 Stability and repeatability tests of SW-Trypsin with BSA

BSA was digested eight times within a month. 
率。选择 3 条零漏切位点肽段 (具体信息见表 3 ), 使用 Pinpoint 软件对数据结果进行分析, 3 条肽段 的总离子流色谱图如图 6 所示。从图 6 中可以看出 蛋白质经 SW-Trypsin 酶解的肽段色谱峰保留时间 与经传统溶液酶解的结果相比基本无偏移。通过计 算得到 3 条肽段回收率(见表 4), 平均肽段回收率 达到 87. 67\%。其中氨基酸序列为 HGTVVLTALGGILK 的肽段回收率相对较低,而该肽段也是所有肽 段中疏水性最强的肽段。该结果也提示了 SWTrypsin 可能对疏水性肽段具有一定的吸附能力。 另外肽段 ALELFR 的回收率大于 $100 \%$,可能是由 于仪器误差、溶液酶解的该肽段存在漏切位点或过 度酶解所致。
表 3 用于考察回收率的特征肽段及其特征离子对

Table 3 Transitions of unique peptides used for evaluating recovery

\begin{tabular}{lccc}
\hline $\begin{array}{c}\text { Amino acid sequence } \\
\text { of peptide }\end{array}$ & $\begin{array}{c}\text { Parent ion } \\
(\mathrm{m} / \boldsymbol{z})\end{array}$ & \multicolumn{2}{c}{$\begin{array}{c}\text { Product ions } \\
(\mathrm{m} / \boldsymbol{z})\end{array}$} \\
\hline VEADIAGHGQEVLIR & 803.931 & 1192.679 & 1079.595 \\
& & 1008.558 & 951.5365 \\
& & 814.4776 & 757.4561 \\
HGTVVLTALGGILK & 689.9244 & 1184.761 & 1083.713 \\
& & 984.6447 & 885.5762 \\
& & 671.4445 & 487.3233 \\
ALELFR & & 430.3018 & 260.1963 \\
& 374.7212 & 322.187 & 564.3135 \\
& & 677.3975 & 435.2709 \\
& & 175.1184 & \\
\hline
\end{tabular}
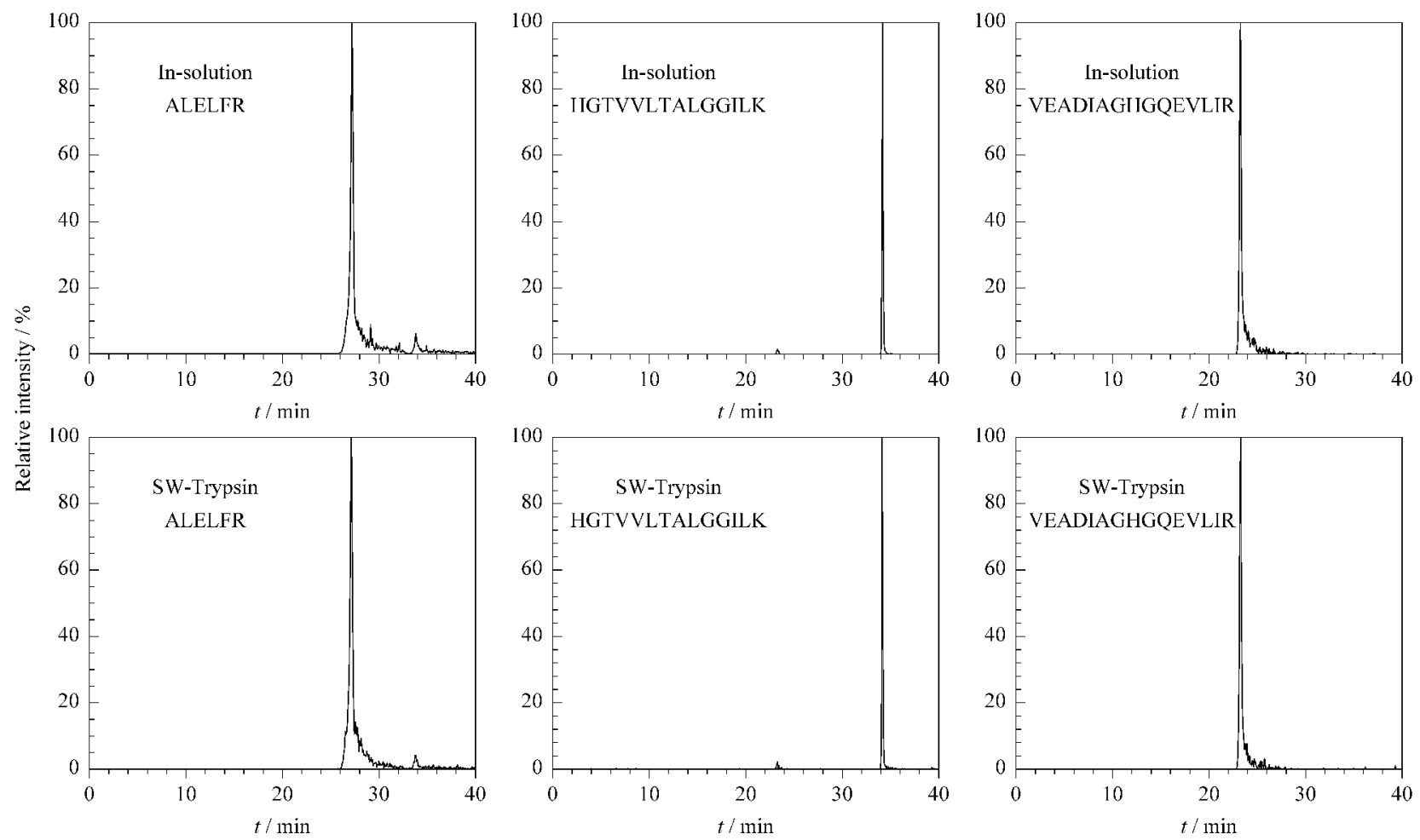

图 6 Pinpoint 软件提取的 3 条肽段的总离子流色谱图

Fig. 6 Total ion chromatograms of three peptides extracted using Pinpoint software

表 4 SW-Trypsin 的回收率考察

Table 4 Recovery of SW-Trypsin

\begin{tabular}{|c|c|c|c|}
\hline \multirow{2}{*}{ Peptide } & \multicolumn{2}{|c|}{ Peak areas } & \multirow{2}{*}{ Recovery $/ \%$} \\
\hline & SW-Trypsin & Free trypsin & \\
\hline ALELFR & $1.895 \times 10^{5}$ & $1.767 \times 10^{5}$ & 107 \\
\hline HGTVVLTALGGILK & $7.694 \times 10^{4}$ & $1.220 \times 10^{5}$ & 63 \\
\hline VEADIAGHGQEVLIR & $5.640 \times 10^{4}$ & $6.092 \times 10^{4}$ & 93 \\
\hline
\end{tabular}

\subsection{SW-Trypsin 用于腾冲嗜热菌蛋白质组鉴定}

使用腾冲嗜热菌作为实际样品, 对 SW-Trypsin 在蛋白质组鉴定中的适用性进行了考察。将腾冲嗜 热菌的全蛋白进行提取及变性后, 取 $50 \mu \mathrm{g}$ 的腾冲
嗜热菌蛋白, 用一根 SW-Trypsin 酶解 $20 \mathrm{~min}$, 另取 $50 \mu \mathrm{g}$ 的腾冲嗜热菌蛋白采用传统溶液酶解方法进 行酶解 $16 \mathrm{~h}$ 。用 RPLC-ESI-LTQ-ICR-FT MS 对两 份酶解样品进行分析。SW-Trypsin 酶解全蛋白的 总离子流图如图 7 所示。使用 $\mathrm{pFind}$ 软件检索腾冲 嗜热菌蛋白质数据库, 结果表明: 经 SW-Trypsin 酶 解后鉴定到 1254 条肽段,归属于 489 个蛋白质。 其中仅 5 条肽段含两个漏切位点, 而含 1 个漏切位 点的肽段也只有 151 条。经传统溶液酶解的样品中 鉴定到 1269 条肽段,归属于 437 个蛋白质。值得 注意的是其中有 49 条肽段含有两个漏切位点, 而含 
有 1 个漏切位点的肽段有 346 条。以上结果证明 SW-Trypsin 具有高效、快速酶解的能力, 可应用于 细胞等复杂体系的全蛋白酶解。

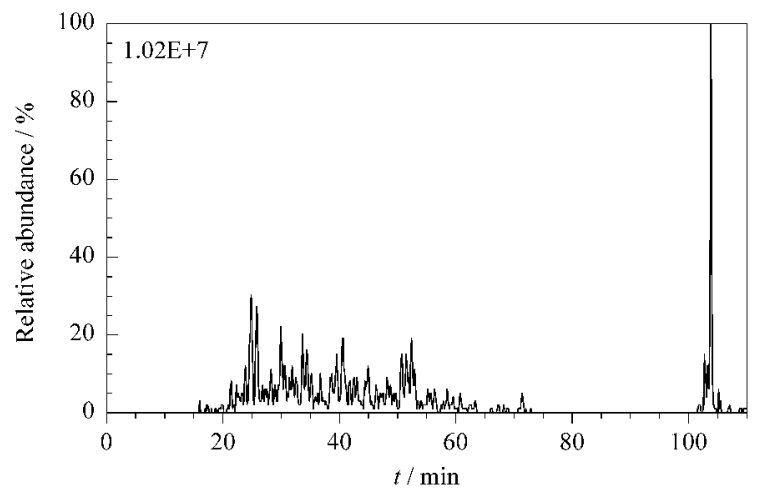

图 7 SW-Trypsin 酶解腾冲嗜热菌全蛋白所得肽段的 基峰色谱图

Fig. 7 Base peak chromatogram of tryptic digests of T. tengcongensis digested by SW-Trypsin

\section{3 结论}

本研究制备了一种新型线毛状聚合物表面修饰 的银丝固定化胰蛋白酶反应器。两种标准蛋白 BSA 和 MYO 经 SW-Trypsin 酶解 $20 \mathrm{~min}$ 后所得的 氨基酸序列覆盖率均好于传统溶液酶解方法, 实验 结果表明该 SW-Trypsin 具有较高的酶解效率。另 外,使用 SW-Trypsin 在一个月内 8 次酶解 BSA 所 得的平均氨基酸序列覆盖率试验和回收率试验结果 表明其具有良好的稳定性和较高的回收率。本研究 还将 SW-Trypsin 成功应用于腾冲嗜热菌蛋白质组 鉴定。加之 SW-Trypsin 容易分离的优点,使其在蛋 白质组学的应用中具有良好的前景。

\section{参考文献:}

[1] Angel T E, Aryal U K, Hengel S M, et al. Chem Soc Rev, $2012,41(10): 3912$

[2] Mann M, Hojrup P, Roepstorff P. Biol Mass Spectrom, $1993,22(6): 338$

[3] Ogorzalek L R R, Hayes R, Yang Y, et al. Int J Mass Spectrom, 2005, 240: 317

[4] McDonald W H, Yates III J R. Curr Opin Mol Ther, 2003, 5 : 302

[5] Xu F, Wang W H, Tan Y J, et al. Anal Chem, 2010, 82 (24) : 10045
[6] Jang H B, Sung H W, Nho S W, et al. Anal Chem, 2011, 83 (5) : 1717

[7] Ren D, Pipes G D, Liu D, et al. Anal Biochem, 2009, 392 (1) : 12

[8] Lazar I M, Ramsey R S, Ramsey J M. Anal Chem, 2001, $73: 1733$

[9] Slysz G W, Schriemer D C. Anal Chem, 2005, 77: 1572

[10 ] Ma J, Liang Z, Qiao X, et al. Anal Chem, 2008, 80: 2949

[11] Ma J, Zhang L, Liang Z, et al. J Sep Sci, 2007, 30 : 3050

[12] Hinterwirth $\mathrm{H}$, Lindner $\mathrm{W}$, Lämmerhofer M. Anal Chim Acta, 2012, 733: 90

[13 ] Mikšík I, Lacinova K, Zmatlíková Z, et al. J Sep Sci, 2012, 35(8) : 994

[14] Kotal M, Srivastava S K, Maiti T K. J Nanosci Nanotechnol, 2011, 11(11) : 10149

[15] Qin W, Song Z, Fan C, et al. Anal Chem. 2012, 84 (7): 3138

[16] Song Z F, Zhang Q L, Zhang Y J, et al. Chinese Journal of Chromatography (宋子凤, 张庆林, 张养军, 等. 色谱), $2012,30(6): 549$

[17] Xu G, Chen X, Hu J, Analyst. 2012, 137: 2757

[18 ] Zhao Y, Liu Y, Lee I, et al. J Biomed Mater Res A, 2012, $100(1): 103$

[19] Liu J, Lin S, Qi D, et al. J Chromatogr A, 2007, 1176(1/) 2) : 169

[20] Nikolic T , Kostic M, Praskalo J, et al. Carb Poly, 2010, 82(3) : 976

[21] Yuan H, Zhang L, Hou C, et al. Anal Chem, 2009, 81 (21) : 8708

[22] Qian K, Wan J, Qiao L, et al. Anal Chem, 2009, 81 (14): 5749

[23] Liang Y, Tao D, Ma J, et al. J Chromatogr A, 2011, 1218 (20) : 2898

[24 ] Hu J W, Shen Y H, Qin W J, et al. Chem J Chinese (胡佳 薇, 申烨华, 秦伟捷, 等. 高等学校化学学报), 2012, 33 (2) : 268

[25] Ma J, Liang Z, Qiao X, et al. Anal Chem, 2008, 80 ( 8 ): 2949

[26] Feng J, Sun M, Xu L, et al. J Chromatogr A, 2011, 1218: 7758

[27] Matyjaszewski K, Xia J. Chem Rev, 2001 , 101: 2921

[28] Tan Y, Yang Q, Sheng D, et al. e-Polymers, 2008, 25: 1

[29] Jones D M, Brown A A, Huck W T S, et al. Langmuir, $2002,18: 1265$

[30 ] Zhang Z, Chen S F, Chang Y, et al. J Phys Chem B, 2006, $110: 10799$

[31 ] Perkins D N, Pappin D J C, Creasy D M, et al. Electrophoresis, 1999, 20(18) : 3551

[32] Li D, Fu Y, Sun R, et al. Bioinformatics, 2005, 21 (13): 3049

[33 ] Wang S, Bao H, Yang P, et al. Anal Chim Acta, 2008, 612 (2) : 182 